The upgraded Pixel Detector of the ATLAS experiment for Run-2 at the Large Hadron Collider

This content has been downloaded from IOPscience. Please scroll down to see the full text.

2016 JINST 11 C02061

(http://iopscience.iop.org/1748-0221/11/02/C02061)

View the table of contents for this issue, or go to the journal homepage for more

Download details:

IP Address: 128.141.192.122

This content was downloaded on 13/02/2017 at 15:19

Please note that terms and conditions apply.

You may also be interested in:

The Pixel Detector of the ATLAS experiment for the Run2 at the Large Hadron Collider

Y. Takubo

The ATLAS Insertable B-Layer: from construction to operation

A. La Rosa

The Pixel Detector of the ATLAS experiment for Run 2 of the Large Hadron Collider

H. Oide

The Pixel Detector of the ATLAS experiment for LHC Run-2

H. Pernegger

The ATLAS Insertable B-Layer project

A Miucci

Overview of the ATLAS insertable B-layer project

F Djama

ATLAS IBL sensor qualification

J Weingarten

"The Read-Out Driver" ROD card for the Insertable B-layer (IBL) detector of the ATLAS experiment: commissioning and upgrade studies for the Pixel Layers 1 and 2 G Balbi, M Bindi, S P Chen et al.

Compute farm software for ATLAS IBL calibration M Bindi, T Flick, J Grosse-Knetter et al. 
$17^{\text {TH }}$ INTERnational Workshop on Radiation Imaging Detectors

28 JUNE - 2 JULY 2015,

DESY, Hamburg, GeRmanY

\section{The upgraded Pixel Detector of the ATLAS experiment for Run-2 at the Large Hadron Collider}

\section{G. Mullier on behalf of the ATLAS collaboration}

AEC-LHEP University of Bern,

Sidlerstrasse 5, 3012 Bern, Switzerland

E-mail: Geoffrey.Mullier@cern.ch

Abstract: The Pixel Detector of the ATLAS experiment has shown excellent performance during the whole Run-1 of the Large Hadron Collider (LHC). Taking advantage of Long Shutdown 1 (LS1) during 2014/2015, the Pixel Detector was brought to surface to equip it with new service panels and to repair modules. The Insertable B-Layer (IBL), a fourth layer of pixel sensors, was installed in-between the existing Pixel Detector and a new beam-pipe at a radius of $3.3 \mathrm{~cm}$. To cope with the high radiation and increased pixel occupancy due to the proximity to the interaction point, two different silicon sensor technologies (planar and 3D) were used and a new readout chip has been designed with CMOS $130 \mathrm{~nm}$ technology with larger area, smaller pixel size and faster readout capability. Dedicated design features in combination with a new composite material were considered and used in order to reduce the material budget of the support structure while keeping the optimal thermo-mechanical performance. An overview of the lessons learned during the IBL project is presented, focusing on the challenges and highlighting the issues met during the production, integration, installation and commissioning phases of the detector. Early performance tests using cosmic and beam data are also presented.

Keywords: Overall mechanics design (support structures and materials, vibration analysis etc); Detector design and construction technologies and materials; Radiation-hard detectors; Radiationhard electronics 


\section{Contents}

1 Introduction 1

2 New service quarter panels and module recovery 1

3 The Insertable B-Layer 2

4 Challenges met during installation and commissioning 3

5 Upgrades during Run-2 5

6 Conclusions 5

\section{Introduction}

The ATLAS Pixel Detector [1] is the innermost part of the ATLAS [2] tracking system and has shown excellent performance during Run-1 of the Large Hadron Collider (LHC) [3]. For the Run-2, the ATLAS Pixel Detector is composed of four barrel layers of pixel sensors and two end caps of three pixel sensor disks each. The outermost three barrel layers and the disks are the originally designed Pixel Detector installed for Run-1. The fourth layer, closest to the beam pipe is called Insertable B-Layer (IBL) and was installed in the Long Shutdown 1 (LS1). Figure 1 shows the ATLAS Pixel Detector without the IBL. The whole system is inside a $2 \mathrm{~T}$ magnetic field generated by a superconducting solenoid and allows charged particle tracking in the pseudo rapidity range of $|\eta|<2.5 .^{1}$ The high granularity and high resolution of the Pixel Detector is essential to the reconstruction of charged particle trajectories, especially the identification of primary and secondary vertices and jets from $b$ quarks.

\section{New service quarter panels and module recovery}

At the beginning of Run-1 the fraction of disabled modules was $2 \%$ and reached $5 \%$ at the end of Run-1. The most common sources of failures were faulty optoboards, electrical-to-optical converter boards, due to failure of the lasers. A complete list of module failures is listed in figure 2 [4]. To allow repairs, the existing Pixel Detector was taken out of ATLAS at the end of Run-1, disassembled and equipped with new services, called new Service Quarter Panels (nSQP) [5]. All cooling and powering connections between the detector and the outside services are provided by the nSQP. There is a total of eight nSQP, four per side of the detector. The installation of new services allowed

${ }^{1}$ ATLAS uses a right-handed coordinate system with its origin at the nominal interaction point (IP) in the centre of the detector and the $z$-axis along the beam pipe. The $x$-axis points from the IP to the centre of the LHC ring, and the $y$-axis points upward. Cylindrical coordinates $(r, \phi)$ are used in the transverse plane, $\phi$ being the azimuthal angle around the $z$-axis. The pseudorapidity is defined in terms of the polar angle $\theta$ as $\eta=-\ln \tan (\theta / 2)$. 


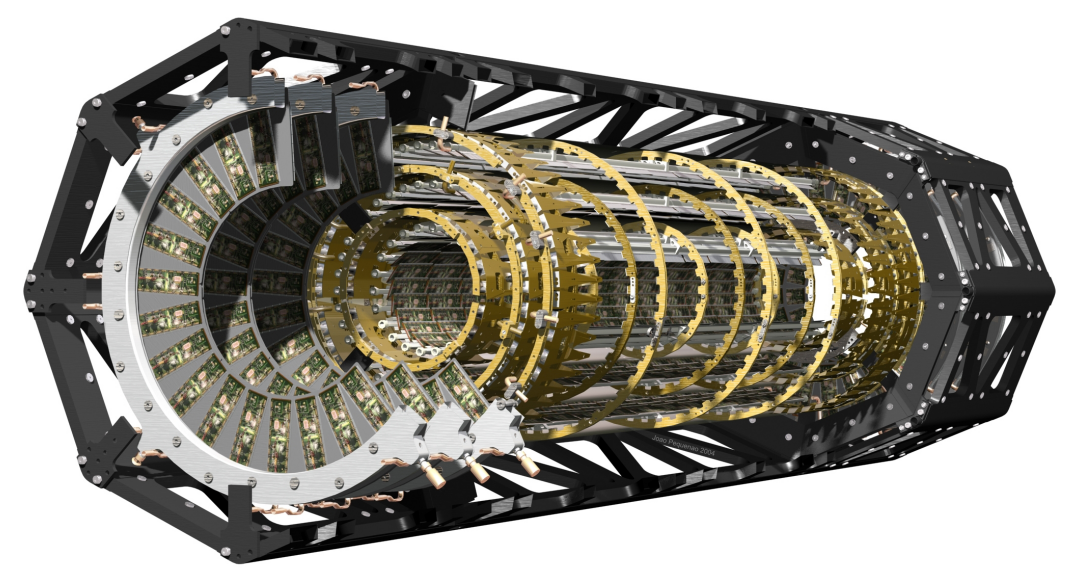

Figure 1. Schematic view of the old pixel detector, not including IBL [6].

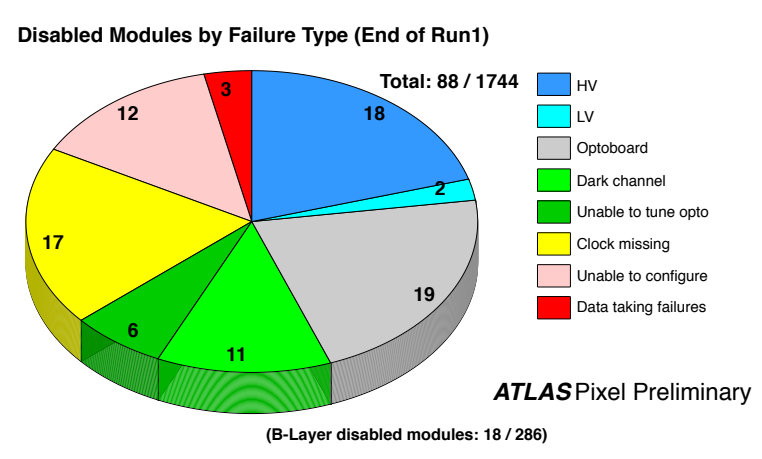

Modules to be disabled (After LS1 Re-installation)

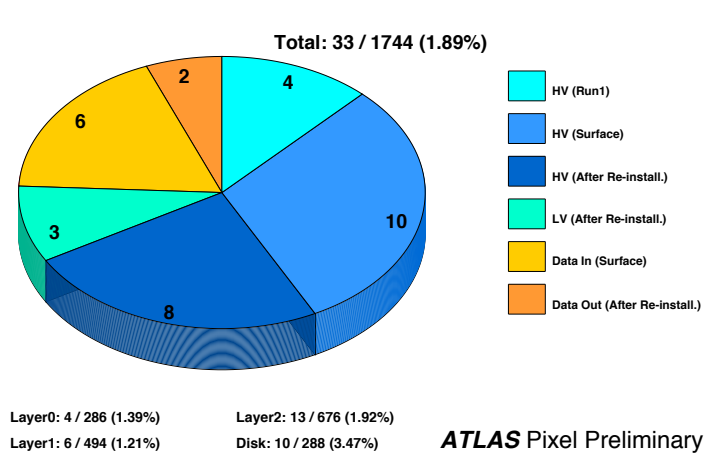

Figure 2. Left: number of disabled modules by failure type for the 5\% disabled pixel modules at the end of Run-1. Right: fraction of the reasons of failure after reinstallation of the Pixel Detector not including the IBL [4].

refurbishment of the existing ones, the upgrade of the optoboards and new optical fibres installation. The optoboards on the nSQP can now be removed from the detector for maintenance.

The number of optical links for the outermost layers has been increased to avoid saturation, even in case of luminosities up to $3 \times 10^{34} \mathrm{~cm}^{-2} \mathrm{~s}^{-1}$. Thanks to the refurbishing and the installation of the nSQP, the number of disabled modules for the original three layer Pixel Detector was brought down to 2\%. Counting IBL, the ATLAS Pixel Detector is now a full four layers, 92 million channels pixel detector with $99.0 \%$ operational fraction.

\section{The Insertable B-Layer}

The IBL [7] was designed to withstand a high luminosity environment and had to overcome technologic challenges set by the high granularity and the required bandwidth of the readout chips. The IBL consists of 14 pixel supporting structures, called staves, each one containing 20 modules with 32 Front-End (FE-I4 [8]) chips. Twelve planar sensors equipped with two front-end chips are located at the centre of each stave and four 3D sensors equipped with single chips at each edge. 
Each pixel has a size of $50 \mu \mathrm{m} \times 250 \mu \mathrm{m}$. The staves are all mounted on the Inner Positioning Tube (IPT) supporting their ends and holding services. The new beam pipe is located inside the IPT and was inserted into the IPT before the IBL integration. The IBL is supported by the IBL Support Tube (IST), installed inside the B-Layer of the Pixel Detector. The IST provides both support and initial position reference of the IBL with respect to the Pixel Detector. Each module is supplied with power and read out via a multilayer flex-circuit, glued to the outside of the stave. The radiation length for the total IBL package is $X / X_{0}=1.9 \%$, with contributions of $X / X_{0}=0.6 \%$ from the modules, of $X / X_{0}=0.6 \%$ from the carbon fibre support staves with cooling and of $X / X_{0}=0.7 \%$ from all support cylinders and services, $X_{0}$ being the radiation length. The most important problems that had to be faced during production and commissioning are discussed in the following section.

\section{Challenges met during installation and commissioning}

Crystalline residues on the flex of the staves, next to the wire bonds were observed during the IBL production. Those residues were due to a corrosion process of the wire-bonds caused by condensation on the stave. This condensation occurred during thermal cycling which is part of the mechanical integrity and quality insurance tests. The wire bonding quality was assessed by mechanical pull tests for the entire production. The corrosion takes place only if condensation occurs, therefore the IBL detector is permanently flushed with nitrogen in the inner detector volume and a careful monitoring of the temperatures and humidity levels is performed. The coldest temperature expected for the IBL is estimated to be $-35^{\circ} \mathrm{C}$, the dew point measured at the exhaust of the IBL volume is below $-70^{\circ} \mathrm{C}$.

During production, wire bonds of the IBL were not encapsulated. The wire bonds transmitting square signals at a rate up to $40 \mathrm{MHz}$ and immersed in the central ATLAS magnetic field can reach an oscillation resonance. This might be the case for certain trigger accept frequencies, corresponding to a certain bunch group configuration from the LHC. Controlled tests [9] showed that for a signal current of $100 \mathrm{~mA}$ in a wire bond of $2.8 \mathrm{~mm}$, the resonant frequency is $9.4 \mathrm{kHz}$. To prevent breaking of wire bonds at resonance frequencies, a fixed frequency trigger veto is applied to filter out problematic trigger signal frequencies. The expected impact on the data taking at LHC design capabilities is estimated to be less than $1 \%$.

The IBL shows significant displacement in $R-\varphi, R$ being the distance measured from the interaction point in the plane perpendicular to the beam pipe and $\varphi$ the angle in the plane perpendicular to the beam pipe. This effect is due to the thermo-mechanical strains on the mechanical structure. The displacement in $\varphi$ is due to the strains put on the central ring holding the IBL that forces a rotation of the staves. The displacement in $R$ is due to the strains put on the staves themselves. This effect has been studied through careful Finite Element simulation of the IBL structure from which a render of the simulation can be seen in figure 3. A correction has been applied based on extensive cosmic ray mapping [10] and precise simulations to quantify the impact on the reconstruction and resolution. This study shows no loss in resolution as can be seen in figure 4 [11]. Since this effect is temperature dependent, a careful monitoring and control of the temperature of the IBL is performed to ensure that the slight deformations of the IBL are acceptable. 


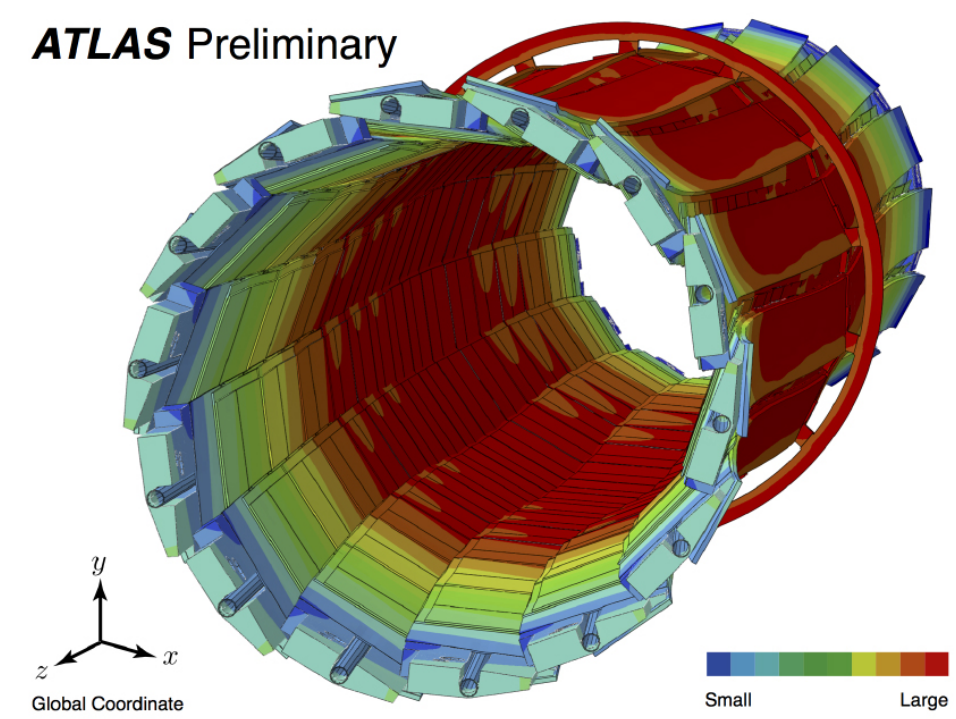

Figure 3. Finite Element simulation at $\Delta T$ of $-60 \mathrm{~K}$ showing the full deformation of the IBL both by the central ring and individual staves. The deformation is magnified 20 times for visualisation [11].

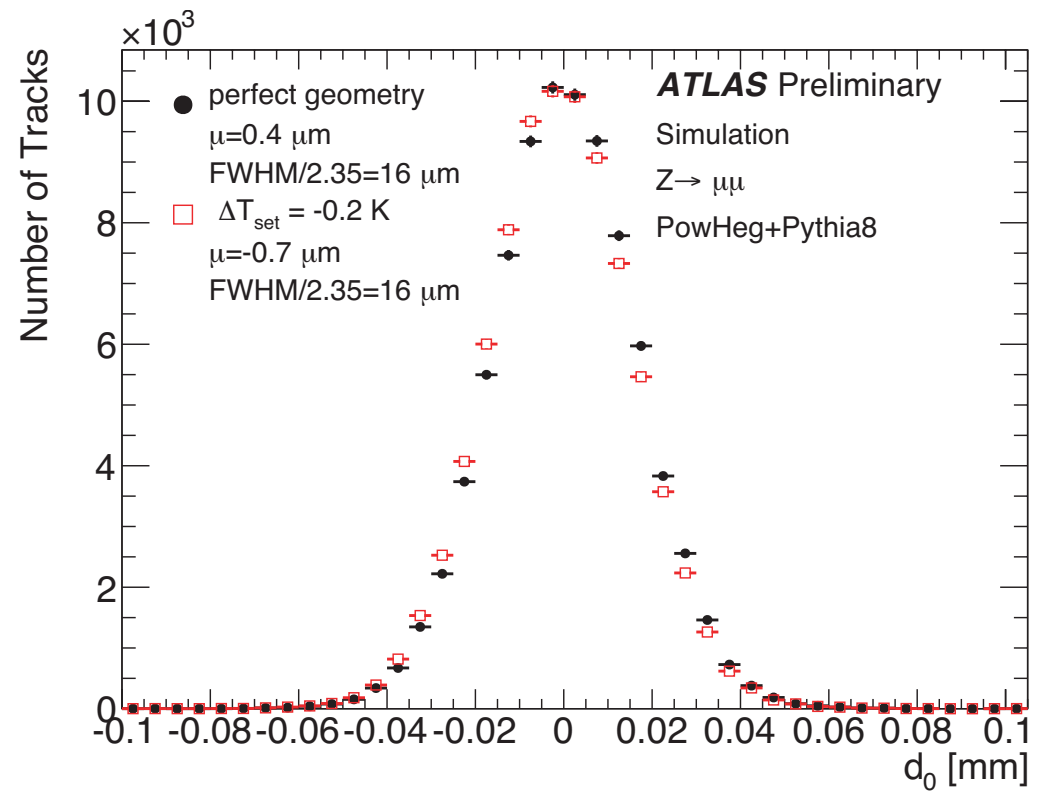

Figure 4. Distribution of the transverse impact parameter $d_{0}$ of charged tracks with respect to the beamspot from $Z \rightarrow \mu^{+}+\mu^{-}$events simulated in $\sqrt{s}=13 \mathrm{TeV}$ pp collisions. The solid circles show the nominal geometry and the open squares show the distorted geometry corresponding to a temperature variation of the IBL at $-0.2 \mathrm{~K}(\approx 2 \mu \mathrm{m}$ of displacement at the center of the stave $)[11]$. 
Table 1. Readout speed of the different pixel detector layers. All the layers use single links, except for the B-Layer that uses two multiplexed $80 \mathrm{Mb} / \mathrm{s}$ links to reach $160 \mathrm{Mb} / \mathrm{s}$.

\begin{tabular}{|ccc|}
\hline Layer & Readout speed & Links used \\
\hline IBL & $160 \mathrm{Mb} / \mathrm{s}$ & Single link \\
B & $160 \mathrm{Mb} / \mathrm{s}$ & Double links \\
1 & $80 \mathrm{Mb} / \mathrm{s}$ & Single link \\
2 & $40 \mathrm{Mb} / \mathrm{s}$ & Single link \\
\hline
\end{tabular}

\section{Upgrades during Run-2}

The occupancy foreseen with the estimated instantaneous luminosity for Run-2 of the LHC will cause saturation of the bandwidth of the data transmission links from the on- to the off-detector electronics, and in the case of the outermost barrel Pixel Detector layers, layer 2 and layer 1, will lead to loss of hit efficiency. First signs of saturation and link errors appeared at the end of Run-1. The current readout speed of the different layers of the Pixel Detector is summarised in table 1. Since a robust readout system was developed for IBL, which is capable of data acquisition at higher bandwidth, the natural solution is to replace the off-detector read out electronics for the Pixel Detector with a system analogous to the one used by IBL. The main challenge of this upgrade project is due to the different transmission encoding used in the IBL with respect to the old pixel modules. The DC non-neutrality is an issue for active optical receivers: commercial plugins, used in the IBL, are designed to be able to automatically tune thresholds by extracting the DC component of the signal. To cope with signals not DC-balanced, new Rx plugins were developed and are currently produced. Installation will take place in January 2016 during the yearly winter LHC shutdown. This will allow the links of layer 2 and 1 to withstand the additional load caused by the increase of data input from those layers.

\section{Conclusions}

The ATLAS Pixel Detector showed excellent performance during Run-1. For Run-2 the Pixel Detector is facing increasing instantaneous luminosity and high pile-up conditions. Primary and secondary vertex reconstruction as well as b-tagging are highly dependent on the good performance of the Pixel Detector. To cope with the pile up and luminosity, the Pixel Detector of ATLAS was upgraded with an additional layer and is now a full four-layer pixel tracking system. Event display with hits from the new layer is shown in figure 5 [12]. New service quarter panels permit an increase in the data transmission bandwidth from the on-detector electronics to the off-detector electronics and in addition a refurbishment of the original detector was performed. The innermost layer, the IBL, shows less than $0.1 \%$ defect channels and can operate with a $1500 e^{-}$threshold. On the outer three layers $98 \%$ of all modules are now fully functional and, after the upgrade of the read-out electronics of the outermost Pixel Detector layers, will be equipped to operate at an instantaneous luminosity of $3 \times 10^{34} \mathrm{~cm}^{-2} \mathrm{~s}^{-1}$ without significant efficiency loss. 

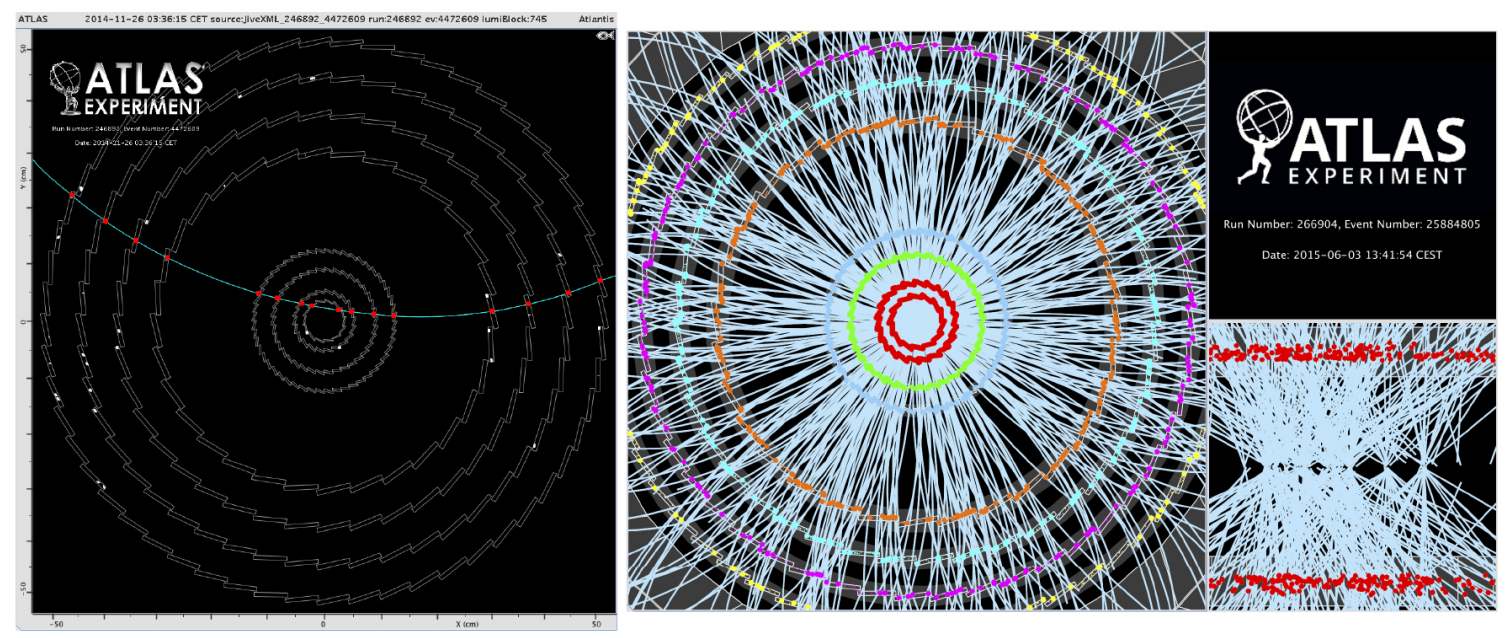

Figure 5. Left: a cosmic ray is shown passing through the IBL, the newly installed pixel layer of the ATLAS Detector, in the presence of a solenoidal magnetic field. The three layers surrounding the IBL are the other layers of the Pixel Detector, and the four outer-most layers seen are the Semiconductor Tracker (SCT). Right: an event display of a proton-proton collision event recorded by ATLAS on 3 June 2015, with the first LHC stable beams at a collision energy of $13 \mathrm{TeV}$. Tracks reconstructed by the tracking detector are shown as light blue lines, and hits in the layers of the silicon tracking detector are shown as coloured filled circles. There is a total of 17 reconstructed collision vertices but they are not all resolvable on the scale of this picture [12].

\section{References}

[1] G. Aad et al., ATLAS Pixel Detector electronics and sensors, 2008 JINST 3 P07007.

[2] ATLAS collaboration, The ATLAS experiment at the CERN Large Hadron Collider, 2008 JINST 3 S08003.

[3] T. Heim, Status and performance of the ATLAS Pixel Detector after 3 years of operation, Nucl. Instrum. Meth. A 765 (2014) 227.

[4] ATLAS collaboration, ATLAS approved pixel plots (2015), https://twiki.cern.ch/twiki/bin/view/AtlasPublic/ApprovedPlotsPixel.

[5] S. Welch and J. Dopke, The ATLAS Pixel nSQP readout chain, ATL-INDET-PROC-2012-018 (2012).

[6] ATLAS collaboration, Computer generated images of the Pixel, part of the ATLAS inner detector, CERN-GE-0803013 (2008).

[7] ATLAS collaboration, ATLAS Insertable B-Layer technical design report, CERN-LHCC-2010-013, ATLAS-TDR-19 (2010).

[8] M. Barbero, The FE-I4 Pixel Readout Chip and the IBL module, ATL-UPGRADE-PROC-2012-001 (2012).

[9] D.A. Feito, A. Honma and B. Mandelli, Studies of IBL wire bonds operation in a ATLAS-like magnetic field, PH-EP-Tech-Note-2015-002 (2015).

[10] ATLAS collaboration, ATLAS inner detector alignment performance with february 2015 cosmic rays data, ATL-PHYS-PUB-2015-009 (2015).

[11] ATLAS collaboration, Study of the mechanical stability of the ATLAS Insertable B-Layer, ATL-INDET-PUB-2015-001 (2015).

[12] ATLAS collaboration, ATLAS event at $13 \mathrm{TeV}$ - first stable beam, 3 June 2015 — run: 266904, evt: 25884805, ATLAS-PHO-Event-2015-016 (2015). 